\title{
Thanking our 2014 peer reviewers
}

Christophe Nicot ${ }^{1 *}$ and Alan Storey ${ }^{2^{*}}$

\section{Contributing reviewers}

The editors of Molecular Cancer would like to thank all the reviewers who contributed to the journal in Volume 13 (2014) by participating in the review process.

Cory Abate Shen

United States of America

Tatjana Adamovic

Sweden

Bharat Aggarwal

United States of America

Tommy Alain

Canada

Arthur Alberts

United States of America

Stefano Alcaro

Italy

Ramon Alemany

Spain

Alain Algazi

United States of America

Lindsey Allan

United Kingdom

Heike Allgayer

Germany

Soner Altiok

United States of America

Noona Ambartsumian

Denmark

Stefan Ambs

United States of America
Hesham Amin

United States of America

Nicola Amodio

Italy

Olga Aprelikova

United States of America

Ulrich Arnold

Germany

Asaithamby Aroumougame

United States of America

Nicola Arrighi

Italy

Michael Ausserlechner

Austria

Farrukh Awan

United States of America

Duncan Ayers

Malta

Hideo Baba

Japan

Ivan Babic

United States of America

Manuela Baccarini

Austria

Serena Rubina Baglio

Italy
Rameshwar Bamezai
India

Dario Barbone

United States of America

Betsy Barnes

United States of America

Mark Barok

Finland

Martin Barr

Ireland

John Barrett

Canada

Monica Bartucci

United States of America

Katia Basso

United States of America

\section{Robert Bast}

United States of America

V. Lokesh Battula

United States of America

James Beattie

United Kingdom

Philippe Becuwe

France

Martin Belinsky

United States of America

\footnotetext{
* Correspondence: molecular-cancer@biomedcentral.com

'Kansas University Medical Center, United States of America, Kansas, USA
}

${ }^{2}$ Oxford, UK 
Brendan Bell

Canada

Marcia Bellon

United States of America

Mariaserena Benassi

Italy

Doris Benbrook

United States of America

Rosaria Benedetti

Italy

Robert Bennett

United States of America

Carmen Berasain

Spain

Michael Berens

United States of America

Michael Bergmann

Austria

Joseph Bertino

United States of America

Giorgio Berton

Italy

\section{Edouard Bertrand}

France

Robert Besch

Germany

Darell Bigner

United States of America

Eric Blair

United Kingdom

Joanne Emily Bluff

United Kingdom

Richard Blumberg

United States of America

Italia Bongarzone

Italy

Maria Carla Bosco

Italy

Hicham Bouhlal

France

Edward Bradley

Canada

Claudio Brancolini

Italy
Randall Brand

United States of America

Walburgis Brenner

Germany

Pnina Brodt

Canada

Laki Buluwela

United Kingdom

Fred Bunz

United States of America

Franco Buonaguro

Italy

Emanuele Buratti

Italy

Andrean Burnett

United States of America

Matthew Burow

United States of America

Ezra Burstein

United States of America

Rita Busuttil

Australia

Qingchun Cai

United States of America

Matthew Caley

United Kingdom

George Calin

United States of America

Daniele Calistri

Italy

Paola Campomenosi

Italy

Carme Camps

United Kingdom

Marco Candeias

Japan

Ida Candiloro

Australia

Anna Rita Cantelmo

Belgium

Qi Cao

United States of America

Bo Cao

United States of America
Guangwen Cao

China

Michele Caraglia

Italy

Matteo Carlino

Australia

Peter Carlsson

Sweden

Maria Carmo-Fonseca

Portugal

Amancio Carnero

Spain

Jason Carroll

United Kingdom

Jon Carthy

Sweden

Lynne Cassimeris

United States of America

Lina Cekaite

Norway

Bo Cen

United States of America

Guralp Ceyhan

Germany

Florence Chainiaux

Belgium

Chiranjib Chakraborty

India

Michael Chan

Taiwan

Stephen Chan

United States of America

Dhyan Chandra

United States of America

Joya Chandra

United States of America

Chawnshang Chang

United States of America

Jang-Yang Chang

Taiwan

Wen-Chang Chang

Taiwan

Pithi Chanvorachote

Thailand 
Sally Chappell

United Kingdom

Srikumar Chellappan

United States of America

Xiaoji Chen

United States of America

Xin Chen

United States of America

Limin Chen

Canada

Rong Chen

United States of America

Yan Chen

China

Yunyun Chen

United States of America

Ann-Lii Cheng

Taiwan

Hua Cheng

United States of America

Liang Cheng

United States of America

Sok Ching Cheong

Malaysia

LUIS Chiva

Spain

William Cho

Hong Kong

Khoi Chu

United States of America

Wen-Ming Chu

United States of America

James Church

United States of America

Chih-Pin Chuu

Taiwan

Bob Clarke

United States of America

Anne-Marie Cleton-Jansen

Netherlands

Nicole Cloonan

Australia

David Cobrinik

United States of America
Yossi Cohen

Israel

Patricia Cohen

United Kingdom

Andrew Colebatch

Australia

Sabine Colnot

France

Paolo Comoglio

Italy

Graham Cook

United Kingdom

John Copeland

Canada

John Copland

United States of America

Pelayo Correa

United States of America

Simona Corso

Italy

Jack Cowland

Denmark

Judy Crabtree

United States of America

Carlo Croce

United States of America

Tim Crook

United Kingdom

Zoran Culig

Austria

Martine Culty

Canada

Artur Czekierdowski

Poland

Min Dai

United States of America

Yan Dai

United States of America

Ichikawa Daisuke

Japan

Sorab Dalal

India

Hien Dang

United States of America
Fanny Daniel

France

Michael Danquah

United States of America

Piyali Dasgupta

United States of America

Kaustubh Datta

United States of America

Ben Davidson

Norway

Kurtis Davies

United States of America

Michael Davies

United States of America

Pieter De Bleser

Belgium

Vittorio de Franciscis

Italy

Steven de Jong

Netherlands

Ivana de la Serna

United States of America

Rainer de Martin

Austria

Giuseppina De Petro

Italy

Anita De Rossi

Italy

Joris de Wit

Belgium

Giannino Del sal

Italy

Dajun Deng

China

Xingming Deng

United States of America

Chadrick Denlinger

United States of America

Paul Dent

United States of America

Punita Dhawan

United States of America

Lucia Di Marcotullio

Italy 
Analisa DiFeo

United States of America

Anna Dimberg

Sweden

Han-Fei Ding

United States of America

Amanda Dixon-McIver

New Zealand

Mustafa Djamgoz

United Kingdom

Yoh Dobashi

Japan

Xiaoqun Dong

United States of America

Yujuan Dong

Hong Kong

Zigang Dong

United States of America

Valérian dormoy

United States of America

Harry Drabkin

United States of America

Liqin Du

United States of America

Wolfgang Dubiel

Germany

Gene Dubowchik

United States of America

Laurence Dubrez

France

Maria Duca

France

Thomas Duchaine

Canada

Stefan Duensing

Germany

Marlène Dufresne

France

Karen Dunphy

United States of America

Joelle Dupont

France

Lee Eckhardt

United States of America
Richard Eckner

United States of America

Rossella Elisei

Italy

Lynne Elmore

United States of America

Georg Enders

Germany

Nuray Erin

Turkey

Stefan Erkeland

Netherlands

Stephen Ethier

United States of America

Muller Fabbri

United States of America

Akos Fabian

Hungary

Isabel Fabregat

Spain

Germana Falcone

Italy

Xiaolong Fan

China

Joachim Fandrey

Germany

Lin Fang

China

Jia Fang

United States of America

Marianne Farnebo

Sweden

Maree Faux

Australia

Eric Fearon

United States of America

Felix Feng

United States of America

Martin Fernandez-Zapico

United States of America

Silvano Ferrini

Italy

Remond J.A. Fijneman

Netherlands
Cristina Fillat

Spain

Paul Fisher

United States of America

Jude Fitzgibbon

United Kingdom

Erik Flemington

United States of America

Abd AlRahman Foda

Egypt

Michael Föller

Germany

C Fordyce

United States of America

Patricia Forgez

France

Al Forrest

Japan

Archa Fox

Australia

Marc Fransen

Belgium

Michael Freeman

United States of America

Leonardo Freire de Lima

Brazil

Steven Frisch

United States of America

Buyin Fu

United States of America

Junya Fujimoto

United States of America

Kiyoko Fukami

Japan

Galina Gabriely

United States of America

Silvia Galardi

Italy

Andrea Galli

Italy

Ian Gallicano

United States of America

Hui Gan

Australia 
Suthakar Ganapathy

United States of America

Allen Gao

United States of America

Yuzhen Gao

China

Giovanni Gaudino

United States of America

Maria Gazouli

Greece

Dirk Geerts

Netherlands

Sarah Gerlo

Belgium

Christian Gespach

France

David Gewirtz

United States of America

Debashis Ghosh

United States of America

Goutam Ghosh Choudhury

United States of America

Sean Gill

Canada

Doron Ginsberg

Israel

Antonio Giordano

United States of America

Jean-Antoine Girault

France

Munirathinam Gnanasekar

United States of America

Katalin Goda

Hungary

Hira Goel

United States of America

Wolfgang H. Goldmann

Germany

David Goltzman

Canada

Vita Golubovskaya

United States of America

Selma de Andrade Gomes

Brazil
Christopher Gondi

United States of America

Lorenza Gonzalez-Mariscal

Mexico

Susana Gonzalo

United States of America

Vidya Gopalakrishnan

United States of America

Margarete Goppelt-Struebe

Germany

Michael Gottesman

United States of America

Susumu Goyama

United States of America

Dinny Graham

Australia

Sheila Graham

United Kingdom

Sveva Grande

Italy

Michael Graner

United States of America

Tim Greten

United States of America

Pat Griffin

United States of America

Thomas Griffith

United States of America

Lyn Griffiths

Australia

Kenneth Grossmann

United States of America

Arnold Grünweller

Germany

Jian Gu

United States of America

Rafael Guerrero-Preston

United States of America

Ghiabe Guibinga

United States of America

Preethi Gunaratne

United States of America

Baoliang Guo

China
Mingzhou Guo

China

Vineet Gupta

United States of America

Katerina Gurova

United States of America

Nikolas Haass

Australia

Trevor Hambley

Australia

Paul Hamel

Canada

Ian Noel Hampson

United Kingdom

Haiyong Han

United States of America

Yuchen han

China

David Harder

United States of America

Johan Hartman

Sweden

Julia Hauer

Germany

Allison Haugrud

United States of America

Petr Hausner

United States of America

Matthew Hayden

United States of America

Nicholas Hayward

Australia

Nicholas Kim Hayward

Australia

Lori Hazlehurst

United States of America

Yulong He

China

Christopher Heaphy

United States of America

Joan Heath

Australia

Olaf Heidenreich

United Kingdom 
Karl Erik Hellstrom

United States of America

Ingegerd Hellstrom

United States of America

Martin Hemler

United States of America

Kyoko Hida

Japan

\section{Martin Higgs}

United Kingdom

Masahiro Higuchi

United States of America

Tetsuro Hirose

Japan

Mary Hitt

Canada

Karin Hochrainer

United States of America

Rob C. Hoeben

Netherlands

Andrea Hoelbl-Kovacic

Austria

Alexander Hoellein

David Hong

United States of America

Liu Hong

China

Wanjin Hong

Singapore

Peter Hordijk

Netherlands

Weihong Hou

United States of America

Gillian Howell

United States of America

Sarah Howie

United Kingdom

Guochang Huang

United States of America

Tony Huang

United States of America

Robert Huebert

United States of America
Kam Hui

Singapore

Joseph Humtsoe

United States of America

Keun Hur

Korea South

Douglas Hurst

United States of America

Antoni Hurtado

Norway

R. Katherine Hyde

United States of America

Inmaculada Ibañez de Caceres

Spain

Zoya Ignatova

Germany

Takashi Ikejima

China

Evgeny Imyanitov

Russian Federation

Hiroyuki Inuzuka

United States of America

John Isaacs

United States of America

Sumiya Ishigami

Japan

Alexander Ishov

United States of America

Maria Izquierdo-Pulido Spain

Meena Jaggi

United States of America

Maneesh Jain

United States of America

Mark Jameson

United States of America

Miriam Jasiulionis

Brazil

Junfang Ji

United States of America

Lijun Jia

China

Bing-Hua Jiang

United States of America
Chenchen Jiang

Australia

Feng Jiang

United States of America

Xuejun Jiang

United States of America

Pengfei Jiang

United States of America

Xi Jiang

United States of America

Xiaohua JIANG

Hong Kong

Lei Jin

Australia

Takashi Joh

Japan

Jeremy Johnson

United States of America

Randy Johnson

United States of America

Frank Jones

United States of America

Edgar Jost

Germany

Anna Margaretha Joubert South Africa

Jingfang Ju

United States of America

Ian Judson

United Kingdom

Kerstin Junker

Germany

Humam Kadara

United States of America

Christoph Kahlert

Germany

Sham Kakar

United States of America

Enikoe Kallay

Austria

Galatea Kallergi

Greece

Dhan Kalvakolanu

United States of America 
Ashish Kamat

United States of America

Bozena Kaminska

Poland

Joseph Kaminski

United States of America

Chunsheng Kang

China

Chunsheng Kang

China

Chi-Dug Kang

Korea South

Ningling Kang

United States of America

Jessica Kao

United States of America

Adam Karpf

United States of America

Santosh Katiyar

United States of America

Masaru Katoh

Japan

Deepak Kaul

India

Sukhwinder Kaur

United States of America

Richard Kefford

Australia

Kornelius Kerl

Germany

Santosh Kesari

United States of America

Ahmad Khalil

United States of America

Hark Kim

Korea South

Hyung Sik Kim

Korea South

Injune Kim

Korea South

Joon Kim

Korea South

Kevin Kim

United States of America
Kyu-Tae KIM

Australia

Jason Kindrachuk

United States of America

Csongor Kiss

Hungary

Uwe Knippschild

Germany

Fumitaka Koga

Japan

Gou Young Koh

Korea South

Eiji Kohmura

Japan

Mikhail Kolonin

United States of America

Masayuki Komada

Japan

Takashi Kondo

Japan

John Koomen

United States of America

Tomasz Kordula

United States of America

Greg Korpanty

Canada

Nina Korzeniewski

Germany

Claudia Kowolik

United States of America

Markus Kretz

Germany

Adam Krieg

United States of America

Natalia Krupenko

United States of America

Georg Krupitza

Austria

Lucia Kucerova

Slovakia

Santosh Kumar

United States of America

Sanjay Kumar

United States of America
Gopal Kundu

India

Siavash Kurdistani

United States of America

Masahiko Kuroda

Japan

Bernhard Kuster

Germany

Vijay Kumar Kutala

India

Yoshikazu Kuwahara

Japan

Young-Guen Kwon

Korea South

Natasha Kyprianou

United States of America

Raymond Lai

Canada

Zhi-Chun Lai

United States of America

Sonia Lain

Sweden

Imayavaramban Lakshmanan

United States of America

Rajkumar Lakshmanaswamy

United States of America

Ola Larsson

United States of America

Pierre Laurent-Puig

France

Gad Lavie

Israel

Brian Law

United States of America

Sean Lawler

United States of America

Yuri Lazebnik

United States of America

Huei Lee

Taiwan

You Mie Lee

Korea South

Po-Huang Lee

Taiwan 
Kin wah Lee

Hong Kong

Susan Lees-Miller

Canada

NL Lehman

United States of America

Ulrich Lehmann

Germany

Dominique Leprince

France

Vera Levina

United States of America

Carmit Levy

Israel

Jian Jian Li

United States of America

Xiang-Ping Li

China

Long-Cheng $\mathrm{Li}$

United States of America

Xiaoling Li

United States of America

Yueming Li

United States of America

Youjun Li

China

Yi Li

United States of America

Min Li

United States of America

Yang Li

China

Jianming Li

China

Zonghai Li

China

Evi Lianidou

Greece

Joshua Liao

United States of America

Jong-Seok Lim

Korea South

Chunru Lin

United States of America
Chin-Yo Lin

United States of America

Dechen Lin

United States of America

Ding-Yen Lin

Taiwan

Zongming Lin

China

Peter Linsley

United States of America

Stanley Lipkowitz

United States of America

Laurie Littlepage

United States of America

Min Liu

China

Bolin Liu

United States of America

Jinsong Liu

United States of America

Ling-Zhi Liu

United States of America

Bo Liu

China

Gongping Liu

China

Zhi-Hua Liu

China

Ying Liu

China

Yangfan Liu

United States of America

Zheng-Gang Liu

United States of America

Zhi-Ren Liu

United States of America

Hui-Wen Lo

United States of America

Kwok Wai Lo

Hong Kong

Joseph Loftus

United States of America

Bal Lokeshwar

United States of America
Anna Lokshin

United States of America

Marc Lopez

France

Haya Lorberboum-Galski

Israel

Fotios Loupakis

Italy

Jose Lozano

Spain

Hua Lu

United States of America

Xiongbin Lu

United States of America

Julian Lum

Canada

Amanda Lund

United States of America

Ji Luo

United States of America

Zhijun Luo

United States of America

Traci Lyons

United States of America

Lukas Mach

Austria

Katerina Machova Polakova

Czech Republic

Marcello Maggiolini

Italy

Kiran Mahajan

United States of America

Pamela Maher

United States of America

Renaud Mahieux

France

Kien Mai

Canada

Mala Maini

United Kingdom

Paraskevi Mallini

United Kingdom

Guidalberto Manfioletti

Italy 


\author{
Koren Mann \\ Canada
}

Sandrine Marchetti

France

Durvanei Maria

Brazil

John Mariadason

Australia

Maria Marino

Italy

John Martens

Netherlands

Pia M Martensen

Denmark

Atsushi Masamune

Japan

Robert Mason

United States of America

Suresh Mathivanan

Australia

Yasunobu Matsuda

Jordan

Isao Matsuura

Taiwan

Michelle Matter

United States of America

Robert Matts

United States of America

Massimiliano Mazzone

Belgium

Dennis McCance

United Kingdom

John McDonald

United States of America

Kerrie McDonald

Australia

Declan McKenna

United Kingdom

Iain McNeish

United Kingdom

Barbara Melendez

Spain

Zhiqiang Meng

China
Laura Mercatali

Italy

Jean-Louis MERLIN

France

Markus Metzler

Germany

Antimo Migliaccio

Italy

Matthew Miller

United States of America

Ian Mills

Norway

Tom Milne

United Kingdom

Toshinari Minamoto

Japan

Nikica Mise

Germany

Akira Miyajima

Japan

Suzanne Miyamoto

United States of America

Yin-Yuan Mo

United States of America

Beverly Mock

United States of America

Faustino Mollinedo

Spain

Hugo Monteiro

Brazil

\section{Rodolfo Montironi \\ Italy}

Richard Moore

United States of America

Hari Narayana Moorthy

Portugal

Mark Morgan

United Kingdom

Pat Morin

United States of America

Sharon Celeste Morley

United States of America

Laura Moro

Italy
David Morris

Australia

Franck Mortreux

France

Justin Mott

United States of America

Mirna Mourtada-Maarabouni

United Kingdom

Caroline Moyret-Lalle

France

Lina Mu

United States of America

Naofumi Mukaida

Japan

Hasan Mukhtar

United States of America

James Mullin

United States of America

Teresita Munoz-Antonia

United States of America

Jordi Muntané

Spain

Satoshi Murata

Japan

Satu Mustjoki

Finland

Joe Mymryk

Canada

Karthikeyan Mythreye

United States of America

Ernest Nadal

United States of America

Alo Nag

India

Sonia Najjar

United States of America

Hiroyasu Nakano

Japan

Ichiro Nakano

United States of America

Harikrishna Nakshatri

United States of America

Kalyan Nannuru

United States of America 


\author{
Christian Nanoff \\ Austria \\ Honami Naora \\ United States of America
}

Aziza Nassar

United States of America

Sathish kumar Natarajan

United States of America

Dirk M. Nettelbeck

Germany

Jochen Neuhaus

Germany

Daniel Neureiter

Austria

Thu Nguyen

United States of America

Bing Ni

China

Christophe Nicot

United States of America

Douglas Noonan

Italy

Susan Nozell

United States of America

Greg Oakley

United States of America

Eva Obermayr

Austria

Josiah Ochieng

United States of America

Takahiro Ochiya

Japan

Yoshinao Oda

Japan

Karin Oechsle

Germany

Yukio Okano

Japan

Barry O'Keefe

United States of America

Denise O'Keefe

United States of America

Soriani Olivier

France

\author{
Ghislain Opdenakker \\ Belgium \\ Véronique Orian-Rousseau \\ Germany \\ Francisco José Ortega \\ Spain \\ Majid Osman \\ Sweden \\ Lindsey Oudijk \\ Netherlands \\ Jaya Padmanabhan \\ United States of America \\ Jihye Paik \\ United States of America \\ José Palacios \\ Spain \\ Tanapat Palaga \\ Thailand
}

Moorthy Palanimuthu Ponnusamy

United States of America

Swati Palit Deb

United States of America

Joanna Pancewicz-Wojtkiewicz Poland

Atanasio Pandiella

Spain

Carlos Panizo

Spain

Benedict Panizza

Australia

Renate Panzer-Grümayer

Austria

Mayra Paolillo

Italy

Anthony Papenfuss

Australia

Luis A Pardo

Germany

Changwon Park

United States of America

Seung Woo Park

Korea South

Won Sang Park

Korea South
Marina Pasca di Magliano

United States of America

Manish Patankar

United States of America

Paola Patrignani

Italy

James Patton

United States of America

Kajsa Paulsson

Sweden

Richard Pearson

Australia

Peter Pelka

Canada

Christopher Penfold

United Kingdom

Guang Peng

United States of America

Guangyong Peng

United States of America

Ignacio Perez de Castro

Spain

Carlos Perez-Stable

United States of America

Guy Perkins

United States of America

Rosario Perona

Spain

Alexander Pertsemlidis

United States of America

Francesca Peruzzi

United States of America

Ulrich Pfeffer

Italy

Francesca Pica

Italy

Julia Pickl

Germany

Smitha Pillai

United States of America

Pascal Pineau

France

Paolo Pinton

Italy 
Ruben Plentz

Germany

Elke Pogge von Strandmann

Germany

Laila Poisson

United States of America

Gretchen Poortinga

Australia

Angel Porgador

Israel

Bellur Prabhakar

United States of America

Vikram Prasad

United States of America

Muriel Priault

France

Esther Priel

Israel

Michele Pritchard

United States of America

Lisa Privette Vinnedge

United States of America

Darwin Prockop

United States of America

Ron Prywes

United States of America

Armin Pycha

Italy

Chao-Nan Qian

China

Wenbin Qian

China

Nancy Raab-Traub

United States of America

Satyanarayana Rachagani

United States of America

Prakash Radhakrishnan

United States of America

Cláudia Rainho

Brazil

Krishnaraj Rajalingam

Germany

Darek Rakus

Poland
Ranju Ralhan

Canada

Sundaram Ramakrishnan

United States of America

Ramani Ramchandran

United States of America

Pranela Rameshwar

United States of America

Joe Ramos

United States of America

Ajay Rana

United States of America

Basabi Rana

United States of America

Katya Ravid

United States of America

Pradip Raychaudhuri

United States of America

Chiara Recchi

United Kingdom

Félix Recillas-Targa

Mexico

Roger Reddel

Australia

Glen Reid

Australia

Milan Reinis

Czech Republic

Jun Ren

United States of America

Peter Revill

Australia

C Patrick Reynolds

United States of America

Vincent Ribrag

France

Carmela Ricciardelli

Australia

Ann Richmond

United States of America

Piotr Rieske

Poland

Markus Riester

United States of America
Natalia Andrea Riobo

United States of America

Arun Rishi

United States of America

Helen Rizos

Australia

Sally Roberts

United Kingdom

Craig Robson

United Kingdom

Sonia Rocha

United Kingdom

Socorro Maria Rodriguez Pinilla

Spain

Sébastien Roger

France

Massimo Roncalli

Italy

Philippe Ronde

France

Zhili Rong

United States of America

Ilse Rooman

Australia

Daniel Rosenberg

United States of America

Robert Roskoski

United States of America

Martin Rottenberg

Sweden

William Roush

United States of America

Carlos Rovira

Sweden

Jeffrey Rubin

United States of America

Mark Russell

United Kingdom

Antonio Russo

Italy

Fred Sablitzky

United Kingdom

Anguraj Sadanandam

United Kingdom 
Cyrus Safinya

United States of America

Susmita Sahoo

United States of America

Neveen Said

United States of America

Akira Saito
Japan

Hiroaki Sakurai

Japan

María Salazar

Spain

Andrea Salmaggi

Italy

Ashok Saluja

United States of America

Rajeev Samant

United States of America

Deepa Sampath

United States of America

Oltea Sampetrean

Japan

David Santamaria

Spain

Giorgio Santoni

Italy

Massimo Santoro

Italy

Takashi Sasayama

Japan

Fuminori Sati

Japan

Shoko Sato

Japan

Yuichiro Sato

Japan

Nicholas Saunders

Australia

Stefania Scala

Italy

Inga-Marie Schaefer

Germany

Liliana Schaefer

Germany
Zachary Schafer

United States of America

Daniela Schilling

Germany

Johannes Schmid

Austria

Laura Schmidt

United States of America

Guenter Schneider

Germany

Sallie Schneider

United States of America

Johannes Schulte

Germany

Gregg Semenza

United States of America

Shantibushan Senapati

India

Peter Senter

United States of America

Parthasarathy Seshacharyulu

United States of America

Claudio Sette

Italy

Mark Shackleton

Australia

Kenneth Shain

United States of America

Ayesha Shajahan-Haq

United States of America

Rajesh Sharan

India

Nima Sharifi

United States of America

Dipali Sharma

United States of America

Hongbing Shen

China

Lalita Shevde-Samant

United States of America

Hubing Shi

United States of America

Jian Shi

United States of America
Lei Shi

China

Masabumi Shibuya

Japan

Vivian Shin

Hong Kong

Takashi Shingu

United States of America

Atsushi Shiozaki

Japan

Adam Shlien

Canada

Robert Shoemaker

United States of America

Viji Shridhar

United States of America

Yongqian Shu

China

Arti Shukla

United States of America

Markus Siegelin

United States of America

Michele Signore

Italy

Robert Silverman

United States of America

Cherie Singer

United States of America

Amar Singh

United States of America

Ajay Singh

United States of America

Dharmendra Singh

United States of America

Pankaj Singh

United States of America

Rakesh Singh

United States of America

Seema Singh

United States of America

Bhawna sirohi

India

Nikolajs Sjakste

Latvia 
Jill Slack-Davis

United States of America

Ana Slipicevic

Norway

DeeDee Smart

United States of America

Degang Song

United States of America

Jianguo Song

China

Guru Sonpavde

United States of America

Richie Soong

Singapore

Rhonda Souza

United States of America

Giulio C. Spagnoli

Switzerland

Riccardo Spizzo

Italy

Sanjay K. Srivastava

United States of America

Peter Stacpoole

United States of America

Olle Stal

Sweden

Daniel Starczynowski

United States of America

Sandra Steffens

Germany

Ulrike Stein

Germany

Carsten Stephan

Germany

Ursula Stochaj

Canada

Christian Stock

Germany

Deborah Stroka

Switzerland

Thorsten Stühmer

Germany

Dwayne Stupack

United States of America
Saraswati Sukumar

United States of America

Yan Sun

China

Luyang sun

China

Robyn Sutherland

Australia

Hiromu Suzuki

Japan

Yoshihiro Suzuki-Karasaki

Japan

Mikael Svensson

Sweden

Elda Tagliabue

Italy

Chiaki Takahashi

Japan

Fuyuhiko Tamanoi

United States of America

Poh Seng Tan

Singapore

Shinji Tanaka

Japan

Reshma Taneja

Singapore

Faqing Tang

China

Takanobu Taniguchi

Japan

Hou-Quan Tao

China

Maria Angels Tapia-Laliena

Germany

Elisa Tassano

Italy

Ada Maria Tata

Italy

Hussein Tawbi

United States of America

Cormac Taylor

Ireland

Kathryn Taylor

United Kingdom
Luba Tchertanov

France

Peter ten Dijke

Netherlands

Vinay Tergaonkar

Singapore

Luigi Terracciano

Switzerland

Stephane Terry

France

George Thalmann

Switzerland

Christoforos Thomas

United States of America

Chenxi Tian

United States of America

Randal Tibbetts

United States of America

Gregory Tietjen

United States of America

Ingeborg Tinhofer

Germany

Verena Maria Tischler

Switzerland

Dean Tolan

United States of America

Ivan Topisirovic

Canada

Nobuyuki Toshikuni

Japan

Sophie Toya

United States of America

Marianna Trakala

Spain

Mark Trifiro

Canada

Tudorita Tumbar

United States of America

Musaffe Tuna

United States of America

Masuko Ushio-Fukai

United States of America

Katsuhiro Uzawa

Japan 
Remco van Doorn

Netherlands

Matthew Vander Heiden

United States of America

Petr Vanhara

Czech Republic

Franz Varga

Austria

Sudhir Varma

United States of America

Edo Vellenga

Netherlands

Pasquale Verde

Italy

Gyorgy Vereb

Hungary

Lars Vereecke

Belgium

Suresh Kumar Verma

United States of America

Alejandro Villagra

United States of America

Carlo Visco

Italy

Rebecca Voltan

Italy

Peter Vrana

United States of America

Paul Wade

United States of America

Pat Wadsworth

United States of America

Bridget Wagner

United States of America

Matthew Wakefield

Australia

Hans Wandall

Denmark

Harold Wanebo

United States of America

Chun-Chao Wang

United States of America

Guoxing Wang

United States of America
Huamin Wang

United States of America

Hongyang Wang

China

Jing Wang

United States of America

Ji Ming Wang

United States of America

Yucai Wang

United States of America

Xiaowei Wang

United States of America

Yan Wang

China

Kishore Wary

United States of America

Toshiki Watanabe

Japan

Stephen Waxman

United States of America

Daniel Weber

Germany

Jun Wei

United States of America

Britta Weigelt

United Kingdom

Michael Weil

United States of America

Andrew Weinberg

United States of America

Jonathan Weitzman

France

Michael Wendt

United States of America

Meir Wetzler

United States of America

Erik Wiemer

Netherlands

David Williams

United States of America

Jason Willis

United States of America

Brice Wilson

United States of America
Ido Wolf

Israel

Georg Wondrak

United States of America

Kwong-Kwok Wong

United States of America

Mark Wong

Australia

Gen Wu

United States of America

Xiangwei Wu

United States of America

Yadi Wu

United States of America

Guo Xiaobo

China

Ke Xu

China

Liang Xu

United States of America

Qilin Xu

United States of America

Nagendra Yadava

United States of America

Nobuo Yaegashi

Japan

Ryuji Yamaguchi

Japan

Munekazu Yamakuchi

Japan

Soichiro Yamamura

United States of America

Min Yan

United States of America

Burton Yang

Canada

Jin-Ming Yang

United States of America

Ruey-Bing Yang

Taiwan

Yong Yang

China

Yanan Yang

United States of America 


\begin{tabular}{|c|c|c|}
\hline Hua Yang & Roza Zakany & Zhihe Zhao \\
\hline China & Hungary & China \\
\hline Ming Yang & Gerard Zambetti & Jun-Nian Zheng \\
\hline China & United States of America & China \\
\hline Young Yang & Hamid Zand & Suting Zheng \\
\hline Korea South & Iran & United States of America \\
\hline Zeng-jie Yang & Mengwei Zang & Wenxin Zheng \\
\hline United States of America & United States of America & United States of America \\
\hline Jason Yap & Giorgio Zauli & Minghao Zhong \\
\hline United Kingdom & Italy & United States of America \\
\hline Chueh-Chuan Yen & Jean-Claude Zenklusen & Weide Zhong \\
\hline Taiwan & United States of America & China \\
\hline Venkat Laxmi Yeruva & Ann Zeuner & Xiaomin Zhong \\
\hline United States of America & Italy & China \\
\hline Junji Yodoi & Jose Zevallos & Chunyan Zhou \\
\hline Japan & United States of America & China \\
\hline Takehiko Yokobori & Dong-Er Zhang & Jianwei Zhou \\
\hline Japan & United States of America & China \\
\hline Takashi Yokota & Jian-Ting Zhang & Binhua Zhou \\
\hline Japan & United States of America & United States of America \\
\hline Yukio Yoneda & Wei Zhang & Yu-Dong Zhou \\
\hline Japan & Singapore & United Kingdom \\
\hline Young Yoo & Wei Zhang & Yi-Hong Zhou \\
\hline Korea South & United States of America & United States of America \\
\hline Go Yoshida & Xu Dong Zhang & Yunli Zhou \\
\hline Japan & Australia & United States of America \\
\hline Kosuke Yoshihara & Xiaohong Zhang & Qinbo Zhou \\
\hline Japan & United States of America & United States of America \\
\hline Liang You & Lingqiang Zhang & ChangQi Zhu \\
\hline United States of America & China & Canada \\
\hline Lawrence Young & Lin Zhang & Jay-Jiguang Zhu \\
\hline United Kingdom & United States of America & United States of America \\
\hline Shi-cang Yu & Wei Zhang & Wei-Guo Zhu \\
\hline China & China & China \\
\hline Xiaozhong Yu & Yongsheng Zhang & Zhengping Zhuang \\
\hline United States of America & China & United States of America \\
\hline Zhang Yu & Jianhua Zhang & Inti Zlobec \\
\hline China & United States of America & Switzerland \\
\hline Zhong Yun & Zhiyong Zhang & Massimo Zollo \\
\hline United States of America & United States of America & Italy \\
\hline Peter Zage & Chen Zhao & Wei-Xing Zong \\
\hline United States of America & United States of America & United States of America \\
\hline Stefan Zahler & Lingyu Zhao & \\
\hline Germany & China & \\
\hline
\end{tabular}

